\title{
Aeroacoustic Investigation of Deployed Spoiler during Steep Approach Landing
}

\author{
Kondwani Kanjere* and Xin Zhang ${ }^{\dagger}$ and Zhiwei $\mathrm{Hu}^{\ddagger}$ and David Angland ${ }^{\S}$ \\ University of Southampton, Southampton, UK
}

\begin{abstract}
A steep approach landing procedure, on a glide slope greater than the conventional 3 degrees glide angle, can be used in order to alleviate noise on the ground, by allowing aircraft to fly higher. Spoilers are then deployed to slow down the aircraft. In this study, a computational aeroacoustic investigation is performed on a two dimensional high-lift system (including slat, main element and flap) in a steep approach landing configuration in order to identify the noise impact of a deployed spoiler. The high-lift model is at an angle of attack of 5 degrees with the slat and the flap deployed at 30 degrees and 38 degrees respectively. The spoiler is deployed at an angle of 20 degrees. The freestream Mach number is $\mathbf{0 . 2 3 5}$, corresponding to a Reynolds number of $5.47 \times 10^{6}$ based on the stowed chord. A hybrid methodology approach involving high order computational fluid dynamics and integral solutions of Ffowcs Williams and Hawkings equation is used to predict the far field noise. The results show that a deployed spoiler changes the flow field around the high-lift devices by reducing the circulation (lift) on all three elements. The slat cove vortex grows in size and the flow separates from the main element leading edge, shedding vortices into the slat gap. The effect of the deployed spoiler on high-lift noise is to increase the level for all radiation angles and all frequencies above $100 \mathrm{~Hz}$. Pressure spectra show that the noise contribution from the spoiler is significant only at low frequencies.
\end{abstract}

\section{Nomenclature}

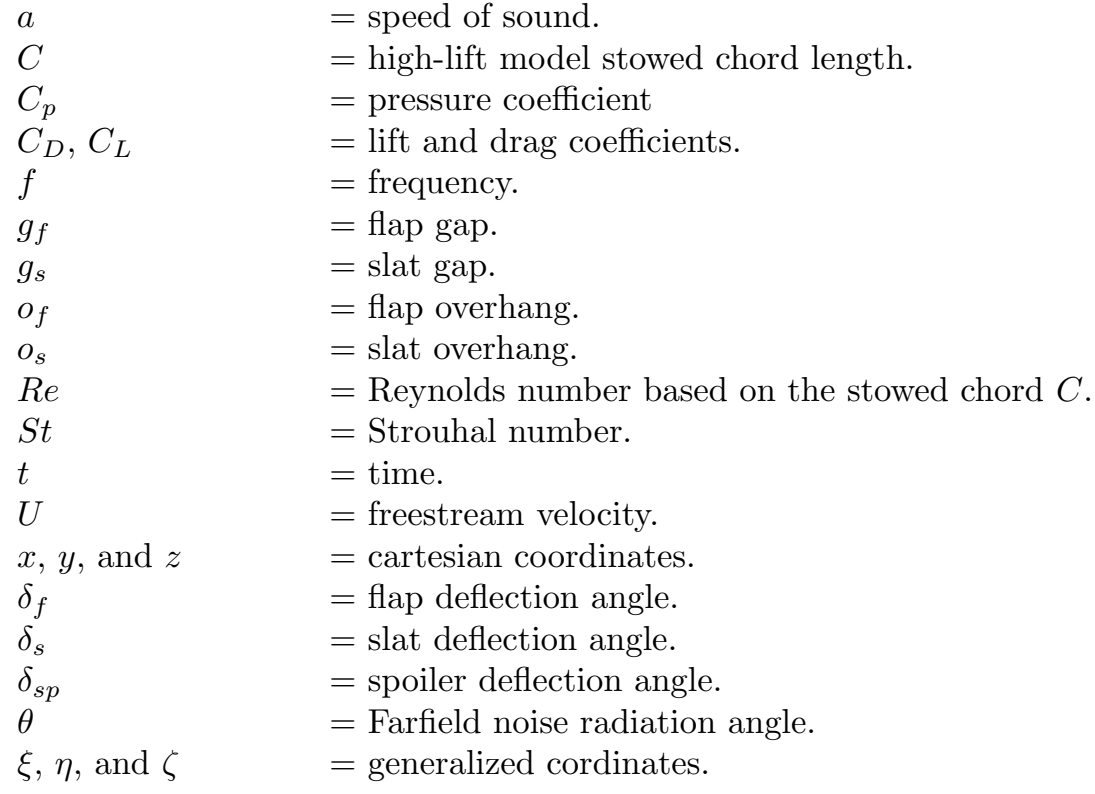

*PhD Student, School of Engineering Sciences, University of Southampton, SO17 1BJ, UK, Student Member AIAA. ${ }^{\dagger}$ Professor, School of Engineering Sciences, University of Southampton, SO17 1BJ, UK, Associate Fellow AIAA.

${ }^{\ddagger}$ Lecturer, School of Engineering Sciences, University of Southampton, SO17 1BJ, UK, Member AIAA.

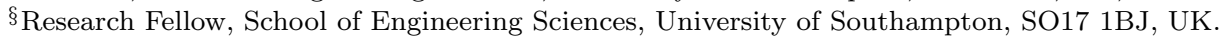


$\omega_{z}$

$=$ spanwise vorticity.

Subscripts

$\overline{()}$

() $R M S$

()$_{\infty}$
$=$ time averaged quantity.

$=$ root mean square quantity.

$=$ freestream quantity.

\section{Introduction}

A DVANCES in low noise high-bypass ratio turbofan engines have resulted in a reduction in jet engine noise such that on approach to landing, when the engines are operating at a low power setting, airframe noise becomes the dominant noise source. Airframe noise is defined as the "non-propulsive noise of an aircraft in flight, due to the airflow around the airframe". The major contributors to airframe noise are landing gears and high-lift devices (HLDs). ${ }^{2}$ Airframe noise causes a lot of annoyance for the communities living near airports. The growth in air traffic at major airports in recent years has resulted in an increase in complaints from the local communities around airports. This has led to governments introducing more stringent noise regulations. Evidently there has been an increased interest in research to understand airframe noise generation mechanisms in order to find ways of reducing aircraft noise impact on communities around airports.

Steep approach landing procedure utilizes a steeper glide slope compared to the conventional 3 degrees glide slope to reduce noise on the ground. By adopting a steeper glide angle, the aircraft flies at higher altitudes over residential areas close to the airport. The engines can be operated at lower power settings because the amount of thrust required to maintain the approach speed is reduced. The combined effect of reduced engine power and greater distance is a reduction in the noise levels on the ground. Steep approach landing procedures are currently operated at several airports e.g. London City airport (LCY). At London City Airport, all aircraft must be capable of making an approach at 5.5 degrees or steeper glide slope in order to operate at the airport. In addition to reducing noise level on the ground, steep approach allows the aircraft to clear obstacles along the flight path e.g. tall buildings around London City airport.

The noise reduction potential of steep approaches has been demonstrated in several studies. Antoine and $\mathrm{Kroo}^{3}$ estimated the noise reduction due to a steep approach at a glide angle of 4.5 degrees to be as much as $7.7 \mathrm{~dB}$ compared to conventional landing at a glide angle of 3 degrees. Clarke et al. ${ }^{4}$ performed flight tests at Louisville International Airport that showed steep approach landing procedures reduced the A-weighted peak noise level along the flight path by 3.9 to $6.5 \mathrm{dBA}$. The study also showed that steep approach reduced the fuel consumption during approach.

For passenger comfort, Dobrzynski ${ }^{5}$ suggests the descent velocity should not exceed $1100 \mathrm{ft} / \mathrm{min}(5.588$ $\mathrm{m} / \mathrm{s}$ ). In FAR/JAR 25.723 the maximum landing descent velocity is $3.66 \mathrm{~m} / \mathrm{s}$ for landing gear stability reasons. In order to meet the descent velocity requirements spoilers can be deployed as airbrakes to slow down the aircraft. A spoiler is a small hinged flat plate that when deflected spoils the flow on the suction surface of a wing. It works by increasing the drag and reducing the lift generated by the wing. Conventionally spoilers are operated on the ground during landing to aid in braking by dumping the lift thereby placing the weight of the aircraft on the landing gear and slowing the aircraft by creating drag. Spoilers are also used to induce rolling moments when operated differentially on the two wings. In steep approach flight tests performed by Clarke et al, ${ }^{4}$ it was noted that pilots used airbrakes (spoilers) to slow down the aircraft on a steep approach. They reported that use of the airbrakes resulted in increased noise levels on the ground. However there was no quantitative data on the noise impact of deployed spoilers. Kipersztok and Sengupta ${ }^{6}$ performed flight test of a 747 aircraft to investigate the noise contribution from different airframe components. The individual component noise contribution was isolated by logarithm subtraction of the noise level of the clean configuration from the configuration with the component deployed. They showed that the noise radiated by the extension of the spoilers caused an increase in level of $3 \mathrm{~dB}$ throughout the entire spectrum (up to $8 \mathrm{kHz}$ ). The noise spectrum of the isolated spoiler was shown to peak at a lower frequency of $80 \mathrm{~Hz}$. The spoiler noise directivity pattern was characterized by radiation into the forward arc similar to the other airframe noise sources (i.e. slat, flap and landing gear). The study was able to quantify the noise impact from the extension of a spoiler. However there is still a lack of understanding of the underlying noise generation mechanisms.

Most of the published literature on spoilers have been on the aerodynamic effects of deployed spoiler in a clean wing configuration (with slat and flap retracted). Experimental studies have shown that the 
flow behind the spoiler is complex involving turbulent separation, ${ }^{7}$ vortex shedding and flow recirculation. ${ }^{8}$ Costes et al. ${ }^{9}$ examined the control surface interaction between a spoiler and flap. The study showed that the spoiler produce greater lift loss for higher flap angles. Computational simulations of spoiler have also focused on the aerodynamic effects, especially the adverse lift effect. The flow field around a deployed spoiler has been simulated by solving the 2D Reynolds-Averaged Navier-Stokes (RANS) equations with different one-equation ${ }^{10}$ and two-equations turbulence models. ${ }^{11}$ The simulations have revealed details of the flow field around the spoiler. At a large enough spoiler deflection angle a vortex forms behind the spoiler. The vortex grows rapidly and induces a strong suction pressure before being shed. The shedding of the vortices corresponds to lift and drag oscillations on the airfoil. Kim and Rho ${ }^{10}$ showed that the vortex shedding frequency decreases with increasing spoiler deflection while the fluctuation amplitude increases with increasing spoiler deflection. The vortex shedding was found to induce a fluctuating pressure field around the whole airfoil. The fluctuating pressure field due to the deployed spoiler is a potential source of noise. In addition the aerodynamic effect of the deployed spoiler on the slat and flap will change the flow dynamics and therefore the noise generation mechanism for the HLDs.

It is clear from the literature reviewed that there is a lack of basic understanding of the noise generation mechanism from HLDs with a deployed spoiler. In this study we perform a high-order CFD simulation of a high-lift wing in steep approach configuration with a deployed spoiler, slat and flap. The two configurations studied are a high-lift model with deployed HLDs and a retracted spoiler, from here on referred to as conventional configuration; and a high-lift model with deployed HLDs and spoiler, from here on referred to as steep configuration. The flow field solutions obtained are then used as inputs to a Ffowcs Williams and Hawkings ${ }^{12}$ (FW-H) acoustic solver used to predict the far field noise spectra and directivity. Detailed comparisons of the near flow and acoustic field and the far field noise spectra are used to identify the impact of the spoiler on HLD noise.

The paper is organized as follows. The numerical methods and the computational grids used in the simulations are outlined in Sec. II. Section III contains the discussion of the flow field and far field noise computation for the conventional and steep configurations. Finally in Sec. IV, summary concluding remarks are given.

\section{Numerical Methods}

\section{II.A. Flow Solver}

The numerical methods used in this study are briefly described in this section. The governing equations are the compressible Navier-Stokes equations.

$$
\frac{\partial \mathbf{Q}}{\partial t}+\frac{\partial \mathbf{F}}{\partial \xi}+\frac{\partial \mathbf{G}}{\partial \eta}+\frac{\partial \mathbf{H}}{\partial \zeta}=\frac{M_{\infty}}{R e}\left[\frac{\partial \mathbf{F}_{v}}{\partial \xi}+\frac{\partial \mathbf{G}_{v}}{\partial \eta}+\frac{\partial \mathbf{H}_{v}}{\partial \zeta}\right]
$$

where $t$ is the time; $\xi, \eta$, and $\zeta$ are the generalized coordinate; $\mathbf{Q}$ is the solution vector; $\mathbf{F}, \mathbf{G}$ and $\mathbf{H}$ are the inviscid flux vectors, and $\mathbf{F}_{v}, \mathbf{G}_{v}$ and $\mathbf{H}_{v}$ are the viscous flux vectors. $M_{\infty}$ is the freestream Mach number and $R e$ is the Reynolds number based on the stowed chord of the high-lift model $C$. The one equation Spalart-Allmaras turbulence model, ${ }^{13}$ from here on referred to as SA model, is used to model the effects of turbulence. Following Khorrami and Lockard ${ }^{14}$ the production term in the SA model equation is switched off in the slat cove region.

The solution is advanced in time by using an implicit Lower-Upper (LU) approximate factorization algorithm employing Newton-like subiterations. Following Jameson ${ }^{15}$, the equations can be written notationally as

$$
\begin{array}{r}
(\mathbf{L}+\mathbf{D}) \mathbf{D}^{-1}(\mathbf{D}+\mathbf{U}) \Delta \mathbf{Q}^{m}=-\frac{3 \mathbf{Q}^{m}-4 \mathbf{Q}^{n}+\mathbf{Q}^{n-1}}{2} \\
-\Delta t D_{\xi 4}\left(\mathbf{F}^{m}-\frac{M_{\infty}}{R e} \mathbf{F}_{v}^{m}\right)-\Delta t D_{\eta 4}\left(\mathbf{G}^{m}-\frac{M_{\infty}}{R e} \mathbf{G}_{v}^{m}\right) \\
-\Delta t D_{\zeta 4}\left(\mathbf{H}^{m}-\frac{M_{\infty}}{R e} \mathbf{H}_{v}^{m}\right),
\end{array}
$$

where $D_{\xi 4}, D_{\eta 4}$ and $D_{\zeta 4}$ represent the 4 th-order optimised prefactored compact difference in the $\xi, \eta$ and $\zeta$ coordinate directions respectively. $\mathbf{L}, \mathbf{U}$ and $\mathbf{D}$ are the LU factorization matrices which are given 
in more detail in Ref. 15. In Eq. 2, a first order backward difference scheme is used to discretize the pseudo-time derivative whereas a second-order central difference scheme is used to discretize the physical time derivative. The spatial derivatives in the implicit segment (left-hand side of Eq. 2) of the algorithm are computed using a second order central scheme whereas the spatial derivatives in the explicit segment (right-hand side of Eq. 2) are computed using the fourth-order optimised prefactored compact scheme of Aschroft and Zhang. ${ }^{16}$ To avoid numerical instability, the solution is filtered at every sub-iteration using a sixth-order nondispersive filter. Second order temporal accuracy is achieved through subiterations to reduce the errors due to factorization, linearization and explicit boundary conditions. Three to five subiterations are found to be sufficient to reduce the residuals by two orders of magnitude. ${ }^{17}$ In this study three sub-iterations are sufficient to reduce the residuals by two orders of magnitude.

The flow solver is based on a finite-difference approach on multiblock structured grids. The code is parallelized using Message-Passing Interface (MPI) to allow efficient communication between connected blocks. A structured grid around a three element high-lift airfoil usually will have block interfaces where there are abrupt changes in the slope of the grid lines. The grid metrics are discontinuous at these points which leads to grid singularity. When high-order finite difference schemes are used to solve the governing equations in generalized coordinates, numerical oscillations can occur at these block interfaces. The characteric interface condition (CIC) of Kim and Lee ${ }^{18}$ is used at block interfaces. The fourth-order optimised prefactored compact scheme ${ }^{16}$ is used in each block: a central difference stencil is applied on the interior nodes from 2 to $N-2$ while on the nodes $1,2, N-1$, and $N$ optimised one-sided difference stencils are used. Thus each block is isolated and the stencils do not cross the block interfaces which eliminates the numerical oscillations. Data between adjacent blocks is exchanged by computing the characteristics across the block boundaries. To prevent the reflection of spurious waves from the edge of the computational domain, explicit buffer zone absorbing boundary conditions are used around the computational domain to damp the conserved variable to freestream values. The effectiveness of the buffer zone boundary condition for preventing reflection of spurious waves was shown by Takeda et al. ${ }^{19}$

A constant non-dimensional time step $\Delta t C / a_{\infty}=4 \times 10^{-4}$, with three Newton-like subiterations is used for the simulation on both configurations. The maximum Courant-Friedrichs-Lewy(CFL) number based on an acoustic velocity $\left(U_{\infty}+a_{\infty}\right)$ is equal to 10 , where $U_{\infty}$ and $a_{\infty}$ are the freestream velocity and speed of sound respectively. The computations are performed on a Linux cluster (Iridis 3) using $642.4 \mathrm{GHz}$ Nehalem CPUs (8 CPUs per node). The cost per time step is 0.32 seconds. The time step corresponds to a sampling rate of $8.5 \mathrm{MHz}$, and the flow through-time based on the stowed chord and the freestream velocity is 10,684 time steps. The flow was run for 850,000 time steps in order to wash out the transients before collecting mean flow statistics.

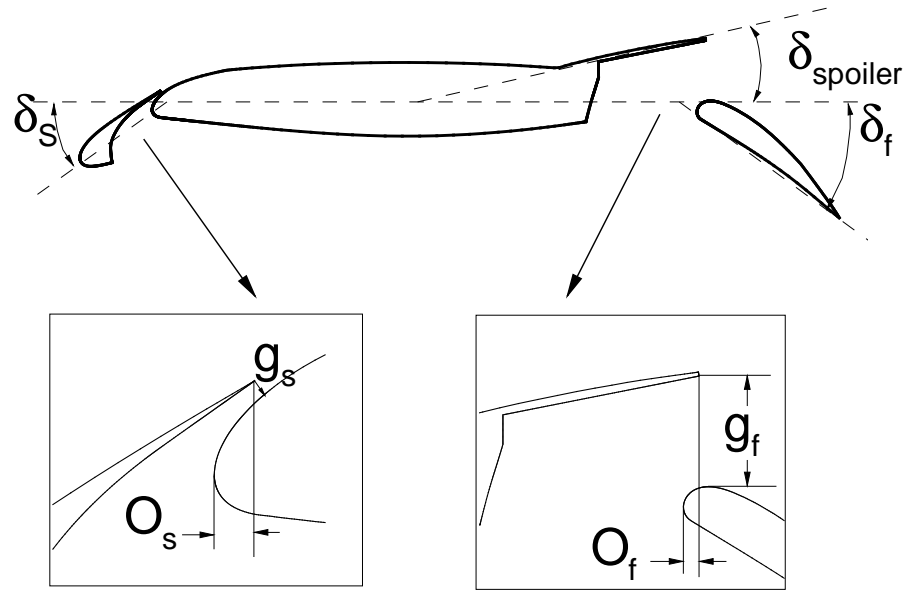

Figure 1. High-lift geometrical settings for the steep configuration. 


\section{II.B. High-Lift Model Geometry and Grid}

The high-lift model comprises of a slat, a main element and a flap. The model has a stowed chord length of $C=1 \mathrm{~m}$. The slat and flap chords correspond to $12 \%$ and $25 \%$ of the stowed chord length respectively. The deflection angles for the slat and the flap are 30 degress and 38 degrees respectively. The steep configuration has a spoiler deployed at an angle of 20 degrees. Figure 1 shows the geometry of the steep high-lift configuration. The geometrical settings for the conventional and the steep configuration are summarized in Table 1. To simulate a typical approach the high-lift model angle of attack for both configurations is set to 5 degrees. The freestream Mach number, $M_{\infty}$ is 0.235 , which corresponds to a Reynolds number of $R e=5.47 \times 10^{6}$ based on the model stowed chord. All the trailing edges are sharp except for the spoiler trailing edge which has a thickness of $0.33 \% C$.

Table 1. Model geometrical settings for the high-lift devices

\begin{tabular}{lcrr}
\hline \hline Parameter & Symbol & Conventional & Steep \\
\hline \hline Reference chord $(\mathrm{m})$ & $C$ & 1.00 & 1.00 \\
Slat angle, degrees & $\delta_{s}$ & 30 & 30 \\
Slat gap, \% C & $g_{s}$ & 0.50 & 0.50 \\
Slat overhang, \% $C$ & $o_{s}$ & 1.22 & 1.22 \\
Flap angle, degrees & $\delta_{f}$ & 38 & 38 \\
Flap gap, \% C & $g_{f}$ & 2.3 & 9.34 \\
Flap overhang, \% C & $o_{f}$ & 0.57 & 1.28 \\
Spoiler angle, degrees & $\delta_{s p}$ & 0 & 20 \\
\hline \hline
\end{tabular}

A structured two dimensional (2D) grid is generated around the high-lift model. The grid generation follows an iterative development path where the grid is refined based on flow field results. The aim is to obtain a grid that could resolve relatively small scale features but has low computational costs. Since the objective of the project is to identify the impact of the deflected spoiler on the high-lift noise, a fine grid resolution is required not only around the spoiler but also around the HLDs.

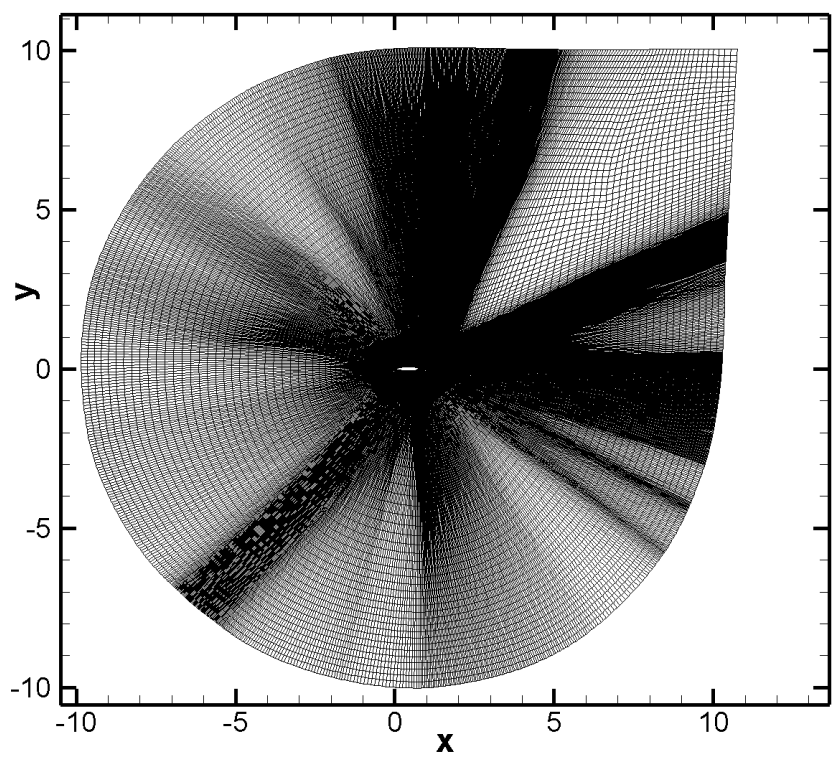

Figure 2. A view of the whole computational domain around the high-lift model.

The computational domain is shown in Fig. 2. For both configurations the domain extends from $-10 C$ to $+10 C$ in the $x$ and $y$ directions. The grid distributions around the slat and the flap for the steep configuration are shown in Fig.3. The grid is clustered in the slat and flap region in order to resolve the unsteady flow. The slat and flap have both sharp trailing edges whereas the spoiler has a blunt trailing edge. Treatment of the 
spoiler blunt trailing edge is crucial to capture the vortex shedding which is reported in the literature. ${ }^{8,10,11}$ Three grid levels are generated for each configuration, a coarse, medium and a fine grid. Successive grids are constructed by grid refinement in region of interest which include the slat cove and gap, flap cove and gap, and the wake of the spoiler and flap (see Fig. 3). The details of the grids are summarised in Table 2. The pressure distribution around the conventional configuration obtained on the coarse, medium and fine grid is shown in Fig. 4. The good agreement between the results obtained on the medium and fine grids demonstration reasonable grid convergence. Similar trends are observed for the steep configuration. The results presented from hereon are the solutions obtained on the fine grid for both configurations.

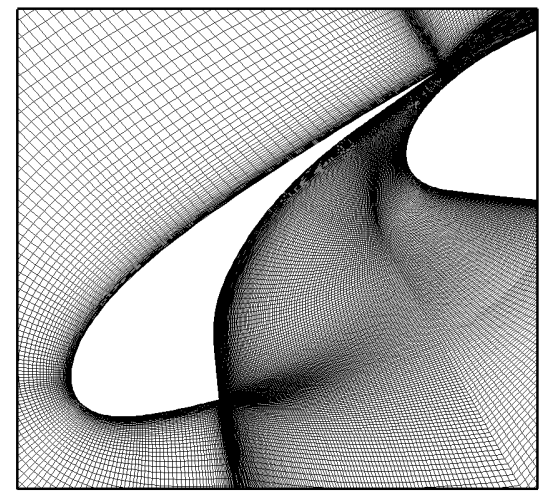

(a) Slat.

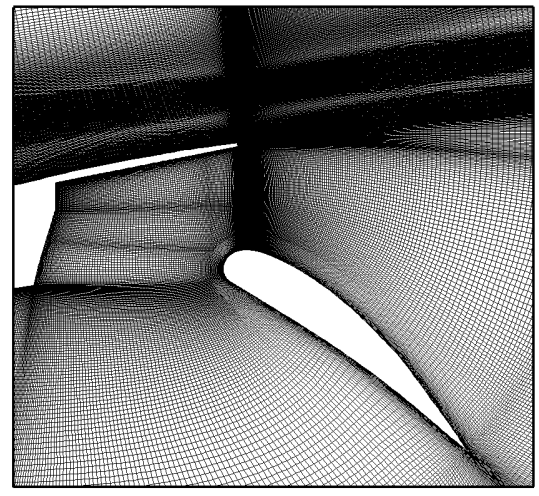

(b) Flap.

Figure 3. Grid distribution in the vicinity of the slat and the flap.

Table 2. Details of the grids for the high-lift model

\begin{tabular}{lrrrrrr}
\hline \hline Parameter & \multicolumn{3}{c}{ Conventional } & \multicolumn{3}{c}{ Steep } \\
& Coarse & Medium & Fine & Coarse & Medium & Fine \\
\hline \hline Total grid points & $2.19 \times 10^{5}$ & $4.26 \times 10^{5}$ & $6.36 \times 10^{5}$ & $2.95 \times 10^{5}$ & $4.33 \times 10^{5}$ & $6.45 \times 10^{5}$ \\
Number of blocks & 128 & 128 & 128 & 134 & 134 & 134 \\
Grid points at spoiler edge & - & - & - & 20 & 30 & 40 \\
\hline \hline
\end{tabular}

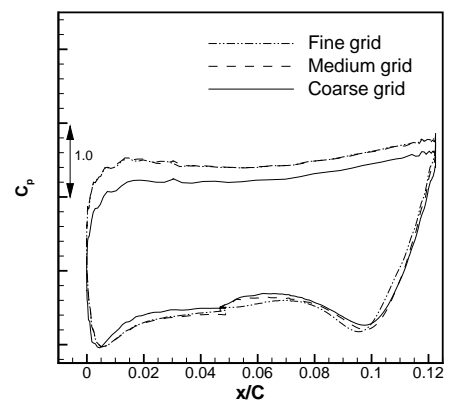

(a) Slat.

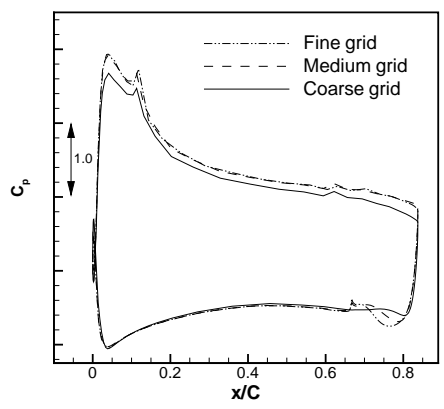

(b) Main element.

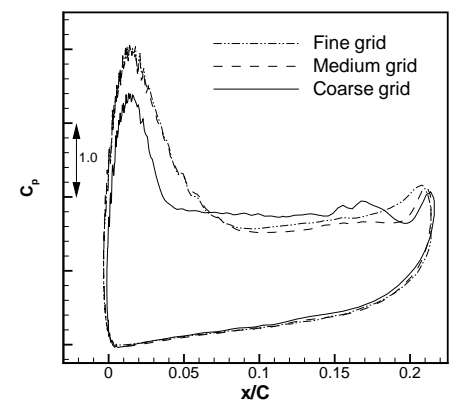

(c) Flap.

Figure 4. Comparison of mean pressure distribution around the conventional configuration obtained using the coarse and fine grid.

The grids are designed to ensure $y^{+} \approx O(1)$ along all solid walls, and approximately 25-35 grid points are located in the boundary layers. A sample of the boundary layer velocity profile on the suction surface of the main element at $x / C=0.78$ is shown in Fig. 5. A comparison is made with a typical turbulent boundary layer velocity profile in the viscous sublayer $\left(y^{+}<5\right)$ and the log-law region $\left(y^{+}>30\right)$. The log-law constants 


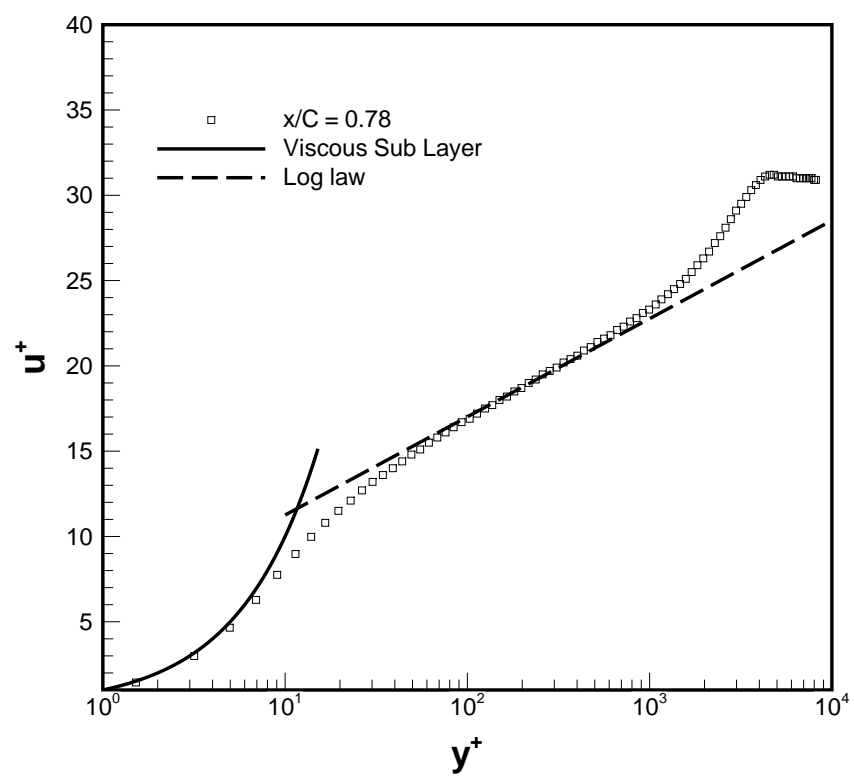

Figure 5. Comparison of the boundary layer velocity profile on main element at $x / C=0.78$.

$\kappa$ and $B$ are set equal to 0.40 and 5.5 respectively. Good agreement between is obtained in both the viscous sublayer and the log-law regions. This shows that the boundary layers are adequately resolved by the fine grids.

\section{II.C. Acoustic Solver}

The far field directivity is computed through an intergral solution of the Ffowcs Williams and Hawkings ${ }^{12}$ (FW-H) equations, based on the formulation 1A of Farassat. ${ }^{20}$ The FW-H solver has been used to compute far field noise from a slat ${ }^{19,21}$ and from an open cavity. ${ }^{22}$ In reaching the solution, only the contributions from the thickness and loading terms are considered whereas the volume source term is neglected. The volume source term consists of quadrupole sources in the flow therefore the volume sources noise scales with the freestream velocity to the power of eight. Since the freestream Mach number is relatively small the contributions from the volume source term is therefore very small hence can be neglected. Two types of integration surfaces are used to compute the far field noise; on-body impermeable surface and an off-body permeable surfaces. Figure 6 shows the on-body and off-body permeable integration surfaces used for the FW-H computations on the steep configuration. The off-body permeable surface contains the slat cove and gap, the flap cove, and the wake of the spoiler and the flap. In addition the off-body permeable surface also contains the boundary layers where it appears to coincide with the solid walls of the three elements.

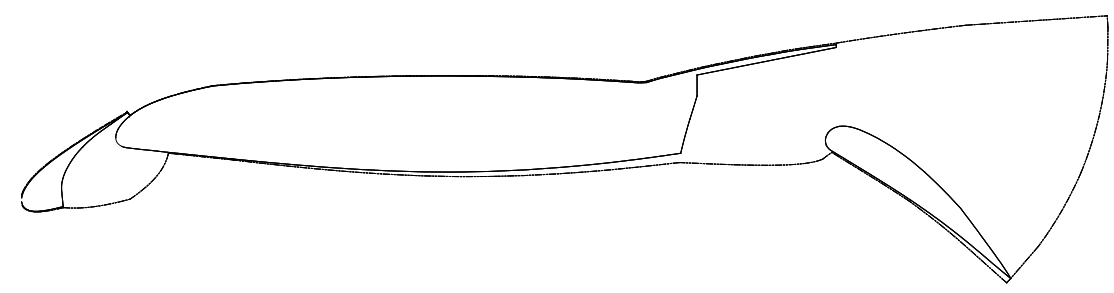

Figure 6. Integration surfaces used for the FW-H calculations. 


\section{Results and Discussion}

\section{III.A. Aerodynamic Flow Field}

Figure 7 compares the time history of the lift and drag coefficients of the steep HLD configuration to the conventional HLD configuration. The coefficients are initially overpredicted however but quickly drop and oscillate around a mean level. reduce quickly but never converge to a steady value. The root mean square (RMS) of the lift and drag coefficients when the spoiler is deployed are $C_{L_{R M S}}=0.1944$ and $C_{D_{R M S}}=0.1085$ where as with the spoiler retracted the lift and drag coefficients are $C_{L_{R M S}}=0.0171$ and $C_{D_{R M S}}=0.0124$. Therefore deflecting the spoiler increases the flow fluctuations by approximately an order of magnitude. The mean lift coefficient is reduced by approximately $60 \%$ and the mean drag cofficient is increased by approximately $72 \%$ when the spoiler is deployed. The fluctuations in the lift and drag coefficients confirm the unsteady nature of the flow around HLDs. The increase in drag is a desirable effect for the purpose of slowing down the aircraft. However the decrease in lift is undesirable since in order to meet landing $C_{L_{\max }}$ requirements, an increase in approach speed and angle of attack is required. Increase in approach speed will result in further increase in noise level.

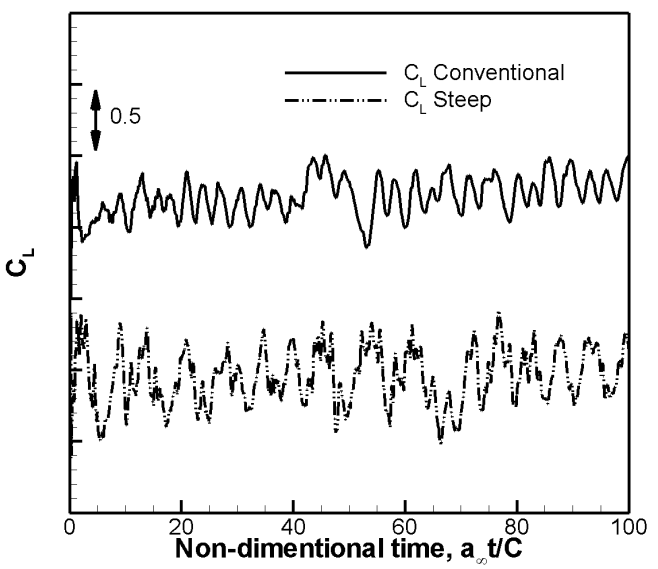

(a) Lift coefficient history.

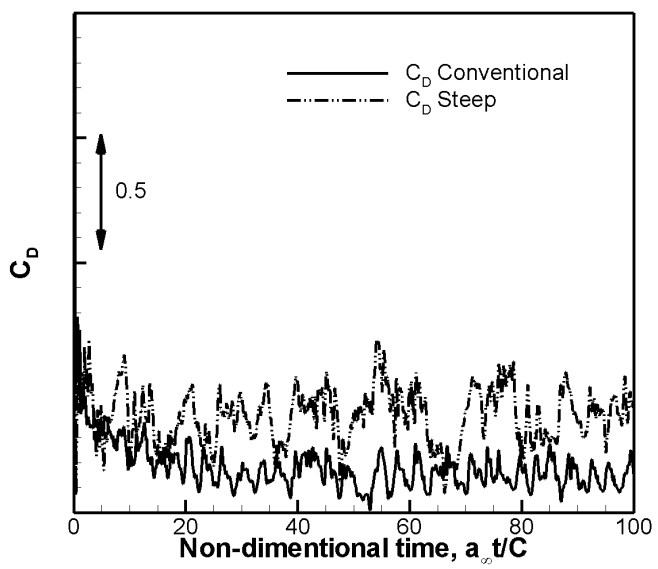

(b) Drag coefficient history.

Figure 7. Comparison of lift and drag coefficient history.

The pressure distribution around the HLDs for the two configurations is shown in Fig. 8. Deploying the spoiler reduces the lift generated by the slat and the main element. On the slat the stagnation point moves to the slat upper surface as a result the flow acceleration occurs on the slat lower surface. On the main element the suction peak level is reduced by half and the stagnation point is moved further downstream. On the flap the pressure plateau after the suction peak corresponds to the large zone of separated flow on the upper surface of the flap.

\section{III.A.1. Instantaneous Flow Field}

The instantaneous flow field around the two HLDs configurations will be analysed in detail in this section. The flow field around the HLDs when the spoiler is deployed is compared to the flow field with the spoiler retracted. A snapshot of the instantaneous spanwise vorticity, $\omega_{z}=\left(\frac{\partial v}{\partial x}-\frac{\partial u}{\partial y}\right)$, around the slat region is shown for both the conventional and steep approach configurations in Figures 9(a) and 9(b) respectively.

In both cases a free shear layer forms at the slat cusp which rolls up into discrete vortices in the slat cove. For the steep configuration the roll-up process is delayed and occurs further downstream from the slat cusp. The vortices take a longer path to the reattachment point in the steep case than in the conventional case. This is due to expansion of the recirculation region within the slat cove when the spoiler is deployed. Deploying the spoiler reduces the circulation (lift) around the entire high-lift wing. As a result the incoming flow incidence at the slat leading edge is reduced. This causes the slat cove vortex region to expand.

At the slat reattachment point the discrete vortices undergo distortions and stretching due to the flow deceleration and subsequent acceleration through the slat gap. In Fig. 9(a), some vortices are trapped in 


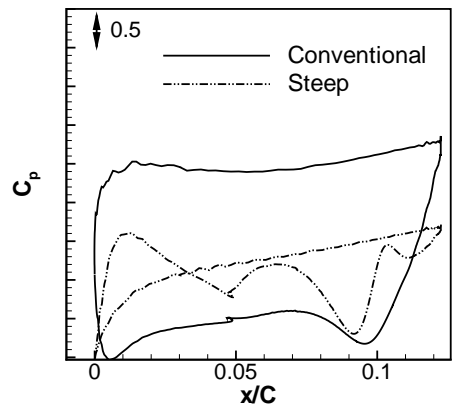

(a) Slat.

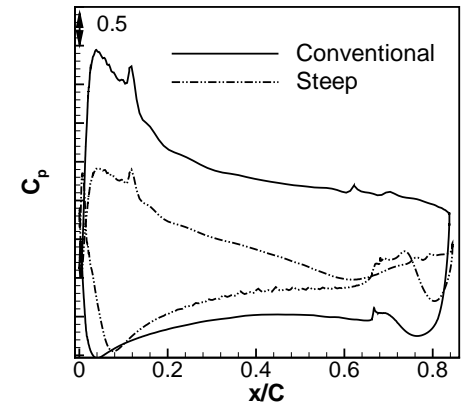

(b) Main element.

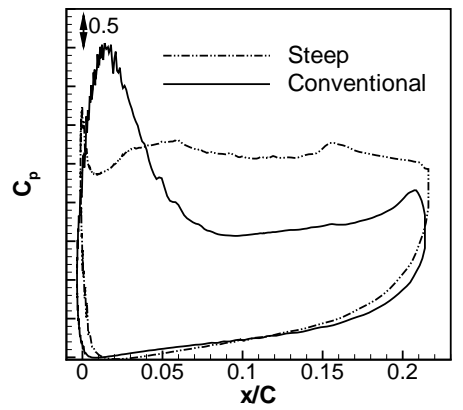

(c) Flap.

Figure 8. Comparison of the mean pressure distribution around the steep (dashdotdot line) and conventional (solid) configuration.

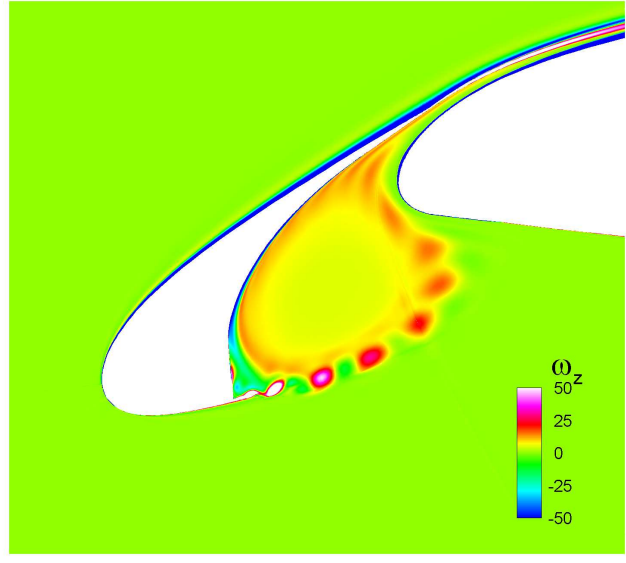

(a) Conventional configuration.

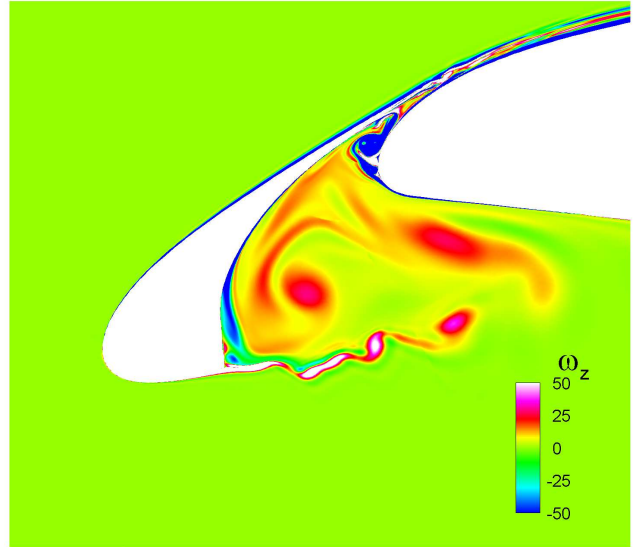

(b) Steep configuration.

Figure 9. Instantaneous non-dimensional spanwise vorticity field around the slat.

the slat cove recirculation flow while some vortices are convected through the slat gap. In the steep case the vortices are only weakly distorted at the reattachment point and most vortices remain trapped in the slat cove. The trapped vortices causes flow separation on the slat lower surface near the slat cusp. The separated shear layer here forms vortices of opposite sign to the vortices from the slat cusp shear layer, thus completing a feedback loop. Similar slat flow dynamics are reported by Khorrami et al. ${ }^{23}$

The reduction in lift around the slat reduces the slat's effectiveness in delaying separation on the main element as a result the flow separates from the main element leading edge as shown in Fig. 9(b). The separated shear layer here quickly rolls up into discrete vortices which are shed through the gap between the slat trailing edge and the main element. The vortex shedding frequency from the main element leading edge is $2.5 \mathrm{kHz}$. The shed vortices interact with the slat lower surface and the slat sharp trailing edge. For the conventional HLD configuration, a small separation bubble forms due to the vortices in the slat cove passing close to the main element leading edge. The separation bubble then convects downstream along the surface of the main element. The effect of this small separation bubble is very small in comparison to the case when the spoiler is deployed such that it is not present in the time-averaged spanwise vorticity distribution. The presence of a turbulent separation bubble at the main leading edge has been previously reported by Terracol et al. ${ }^{24}$ They performed a hybrid RANS/LES simulation of the flow around a three dimensional (3D) high-lift model with the slat and flap deployed. In comparison with a RANS computation the hybrid RANS/LES computation captured the presence of a separation bubble at the leading edge of the main element which caused a small loss of lift.

The instantaneous spanwise vorticity, $\omega_{z}$, around the flap for the conventional and steep HLDs configu- 


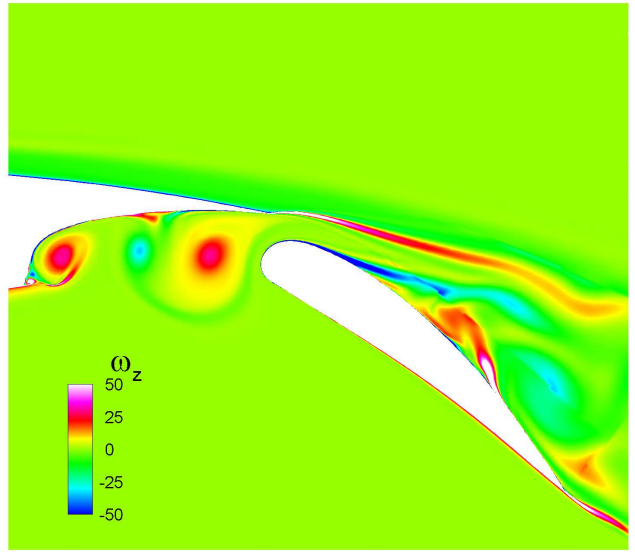

(a) Conventional configuration.

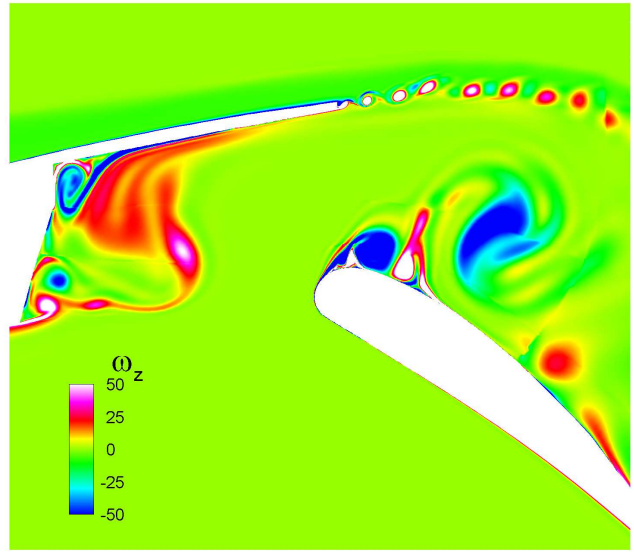

(b) Steep configuration.

Figure 10. Instantaneous non-dimensional spanwise vorticity field around the flap and spoiler.

rations are shown in Figures 10(a) and 10(b) respectively. The deployed spoiler creates a low pressure region above the flap leading edge. This creates an adverse pressure gradient which causes the flow to separate early from the flap leading edge to form a large recirculation region above the flap. For the conventional case, flow acceleration through the small flap gap re-energises the boundary layer, therefore the flow separates further downstream along the flap at approximately $50 \%$ flap chord.

At the spoiler trailing edge, vortex shedding occurs at a frequency of $4.3 \mathrm{kHz}$ which corresponds to a Strouhal number of $S t=0.16$ based on the spoiler trailing edge thickness. The shed vortices are entrained into the recirculating flow above the flap upper surface. At the flap trailing edge the separated shear layer rolls up into discrete vortices with positive vorticity. The recirculation region above the flap is a source of negative vortices. The flow interactions discussed above result in shedding of large vortical structures in the wake of the steep HLD configuration. In contrast for the conventional HLD configuration, the separated flow forms a small recirculation region on the upper surface of the flap. The wake behind the conventional HLDs is narrower and dominated by relatively small vortical structures in comparison with the steep HLD configuration. Pressure monitors are positioned downstream of the flap trailing edge to measure the pressure fluctuations in the wake. The frequency of the vortex shedding from the flap for the conventional HLD configuration is $f=103 \mathrm{~Hz}$ whereas the vortex shedding frequency from the steep HLD configuration is $f=52 \mathrm{~Hz}$. Assuming the vortex shedding occurs at a given Strouhal number then reducing the characteristic length by half will double the vortex shedding frequency. Therefore its argued that the important length scale for the vortex shedding from the flap trailing edge is the length of the recirculation zone above the flap upper surface. For the steep HLD configuration, the length of the recirculation zone is approximately the flap chord, $L=0.247 C$. Thus the Strouhal number based on $L$ is $S t=0.160$. For the conventional HLD configuration, the length of the recirculation zone is approximately half the flap chord, thus the Strouhal number is $S t=0.159$ based on the characteristic length $L=0.1235 C$.

Figure 10 also highlights the unsteady flow field in the flap cove. For the conventional HLD configuration the shear layer separates from the flap cusp and rolls up into discrete vortices. The vortex grows in size with the addition of fresh separated shear layer from the flap cusp until later it detaches and convects downstream into the flap gap. Similar flow dynamics are observed from the steep HLD configuration. The vortex however, is stretched at it convects towards the spoiler trailing edge. The interaction of the vortices with the sharp edges of the main element and the spoiler is a potential source of noise. The shedding of coherent vortices from the flap cove of a three-element high-lift aerofoil has been previous reported by Takeda et al. ${ }^{25}$ They preformed Laser Doppler Anemometry (LDA) and Particle Image Velocimetry (PIV) measurements of the flow around the flap cove. They also found that the wake of the main element trailing edge resembles a mixing layer with little evidence of vortex shedding. Figure 10(a) shows similar flow feature, a free shear layer emanates from the main element trailing edge. 


\section{III.A.2. Time Averaged Flow Field}

In this section the mean flow field around the HLDs is analysed. Figures 11(a) and 11(b) shows the timeaveraged velocity field and streamlines around the slat for the conventional and steep configuration respectively. The streamlines clearly shows the effect of deploying the spoiler on the flow around the slat. For the steep case, the angle of incidence of the incoming flow is reduced as a result of the decreased circulation caused by the spoiler. The stagnation point has moved to the suction surface of the slat. The recirculation region in the slat cove grows in size when the spoiler is deployed. In Fig. 11(b) streamlines also show the flow separation at the main element leading edge which occurs when the spoiler is deployed and the downstream movement of the stagnation point on the main element. The velocity magnitude contours illustrate a reduction in the flow acceleration on the slat suction side and the main element leading edge. This agrees with the observed pressure distributions in Fig. 8. The time averaged non-dimensional spanwise vorticity field

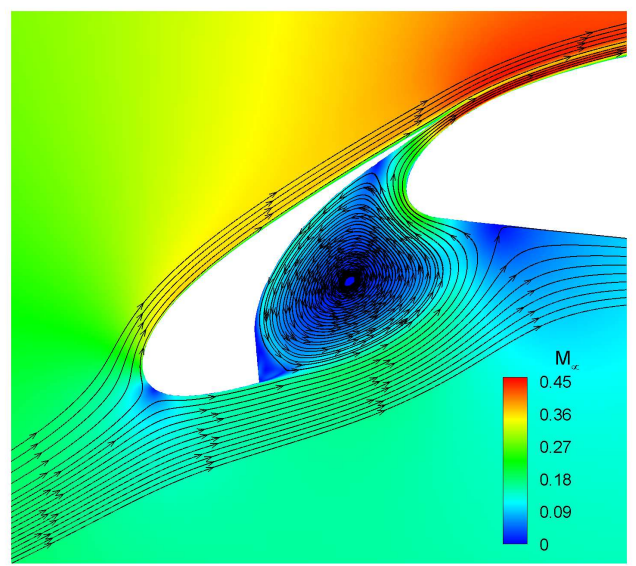

(a) Conventional configuration.

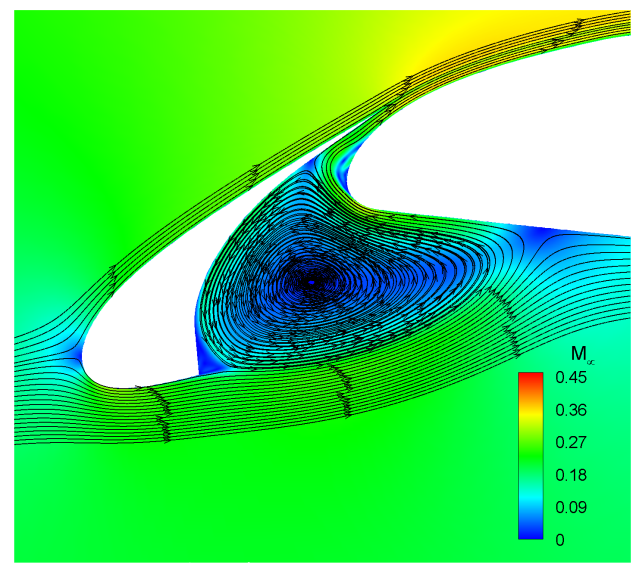

(b) Steep configuration.

Figure 11. Contours of mean velocity magnitude and flow streamlines around the slat.

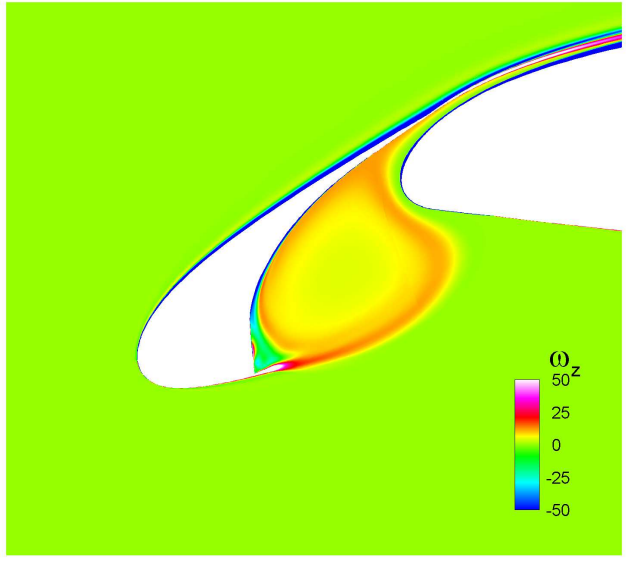

(a) Conventional configuration.

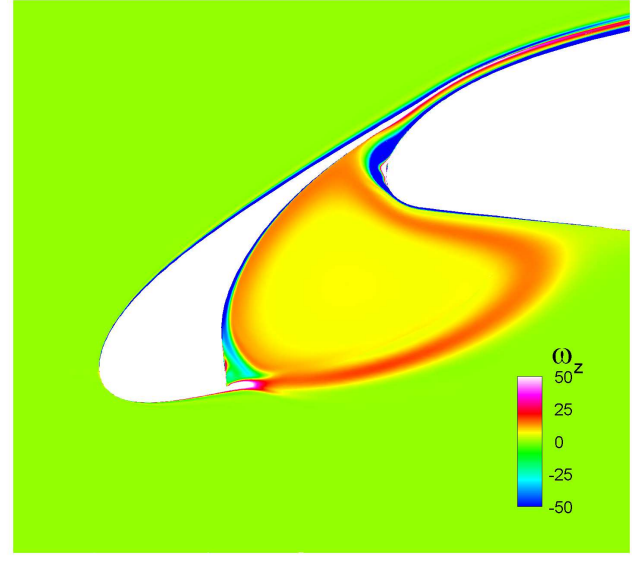

(b) Steep configuration.

Figure 12. Time averaged non-dimensional spanwise vorticity field around the slat.

around the slat for the conventional and steep configuration are shown in Fig. 12(a) and 12(b) respectively. The trajectory of the vortices from the slat cusp to the reattchment point is clearly shown. For the steep case, the path of the free shear layer is longer and encloses a larger region. The vorticity levels remain relatively high from slat cusp to reattachment for the steep case in comparison with the convectional case. A thicker free shear layer bounds the slat cove flow for the steep case. This supports the observation from 
the instantaneous vorticity field that shows larger and more-energetic vortices for the steep case than the conventional case. The time averaged flow field also shows the free shear layer with negative vorticity that separates from the main element leading edge.

\section{III.B. Acoustic Field}

\section{III.B.1. Near Field Propagation}

Snap shots of the instantaneous dilatation field, $\nabla \cdot \mathrm{U}=\left(\frac{\partial u}{\partial x}+\frac{\partial v}{\partial y}\right)$, around the HLD configurations are given in Fig. 13. According to the continuity equation, dilatation is the time rate of change of density (or pressure in an isentropic acoustic field). ${ }^{26}$ Thus the quantity of dilatation is suitable for showing acoustic wave propagation. Figure 13(a) shows the sound propagation from the conventional HLD configuration. The

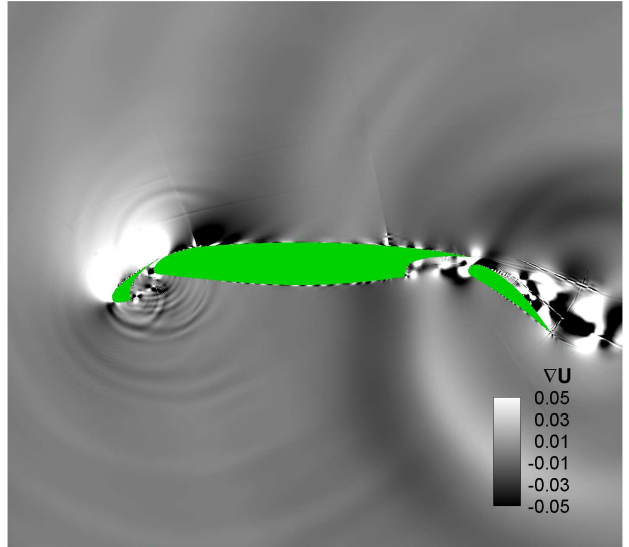

(a) Conventional configuration.

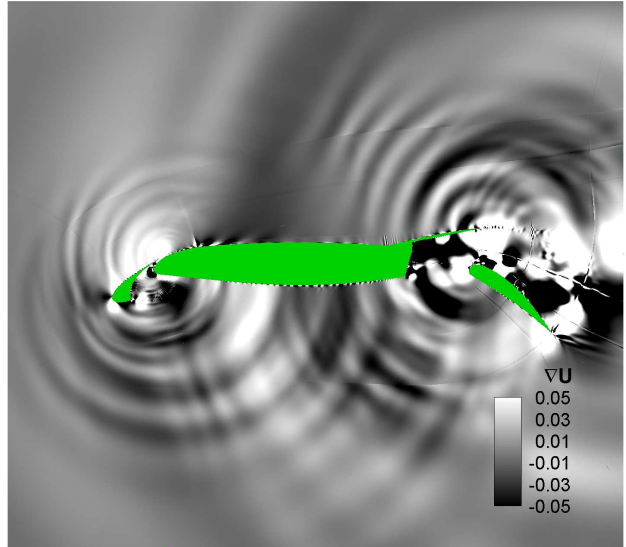

(b) Steep configuration.

Figure 13. Instantaneous dilatation field around the HLDs (lighter contours: $\nabla \cdot \mathrm{U}=-0.05$ and darker contours: $\nabla \cdot \mathrm{U}=+0.05)$.

slat region is clearly a source of noise for this configuration. A close scrutiny of time sequence dilatation plots around the slat showed the noise is generated at the slat trailing edge. The emission of acoustic waves coincided with the shedding of vortices into the slat gap from the slat cove flow. Thus the results suggest a potential noise generation mechanism at the slat is the flow-surface interaction of the vortices with the sharp trailing edge. Analysis also revealed the wing trailing edge to be a source of low frequency sound. The instantaneous flow field in the flap cove show showedthe separated shear layer rolls up into a large vortex that is shed into the flap gap. The interaction of the vortices with the main element sharp edge as they pass through the flap gap is responsible for the observed low frequency sound from the main element trailing edge. The vortex shedding from the flap trailing edge is also identified as a source of low frequency sound which can be seen in Fig. 13(a) radiating from the bottom surface of the flap.

As highlighted before deploying the spoiler causes the expansion of the slat cove recirculation region and flow separation at the main element leading edge. In Fig. 13(b) noise is seen to radiate from the slat region. Again a main source of noise is found to be the slat trailing edge. The flow separation at the main element leading edge sheds vortices into the slat gap past the slat trailing edge. The interaction of the vortices with the sharp edge produces noise. The level of the sound from the slat in steep configuration is higher compared to the conventional configuration. This can be attributed to the close proximity of the main element leading edge to the slat trailing edge. Therefore the vortices diffuse and reduce in strength before passing by the slat trailing edge. The acoustic waves are observed to reflect and diffract around the slat cusp as they propagate through the slat cove towards the ground. At the spoiler trailing edge, noise radiation due to the vortex shedding is observed. This is the direct noise generation mechanism for a deployed spoiler. Similar to the conventional configuration the vortex shedding at the flap trailing edge is a source of low frequency sound. 


\section{III.B.2. Far-field Radiation}

The far field acoustics are computed through an integral solution of the FW-H equation. The unsteady flow data collected on the on-body and off-body permeable integration surfaces are used as input to the FW-H acoustic solver. The unsteady flow data is collected on the integration surface after the initial transient flow solution had passed. After which the data is collected every 25 time steps, corresponding to a sampling rate of $68 \mathrm{kHz}$ for a dimensional time step of $\Delta t=1.1755 \times 10^{-6}$ seconds. In total 10,000 time steps are collected, which are divided into 3 blocks of 4096 samples with a $50 \%$ overlap. A hanning windowing function is used on each block before performing a Fast Fourier Transform (FFT). The sample size give a frequency resolution of $41 \mathrm{~Hz}$.

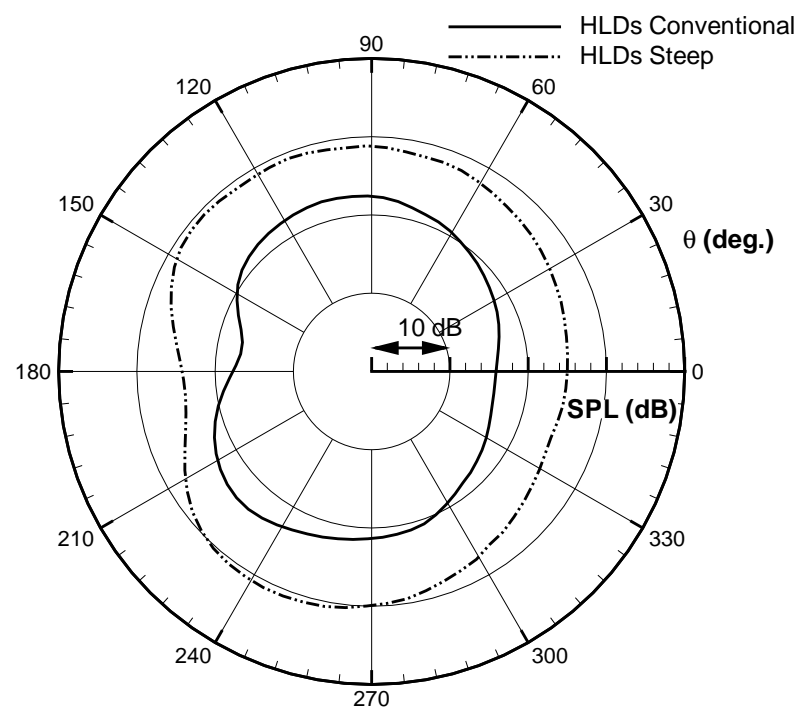

Figure 14. Comparison of far field noise directivity from on-body integration surfaces.

The FW-H solver only computes far field noise from a 3D integration surface. Therefore in order to compute far field noise from a 2D near field CFD dataset, the 2D integration surface is extruded in the spanwise direction. The assumption is that the flow is perfectly correlated over the spanwise length. Takeda et al. ${ }^{19}$ measured the spanwise correlation length on a $\frac{1}{5}$ th scale National high-lift (NHL) model to be equal to $15 \%$ of the model chord length. Based on this measurement the spanwise length used is $0.15 C$. Each integration surface is divided into panels and the flow data is averaged over each panel center. Observers are placed at a distance 100 chord lengths away from the HLD model. The contributions from integration surface panels are summed up for each observer location to obtain the far field pressure. The observer angle, $\theta$, is defined such that zero degrees is pointing in the downstream direction and increases in the counter-clockwise direction. The far field noise prediction from the off-body permeable integration surface was found to be inconsistent with the prediction from the on-body integration surface. Casper et $\mathrm{al}^{27}$ found that the wake passing through the off-body permeable integration surface introduced error in the far field noise predictions. In the current study, attempts to eliminate the effect of the wake passing through the off-body permeable integration surface by using an open integration surface constructed with the wake portion removed was unsucessfull. The far field noise prediction presented in this paper are therefore only from the on-body FW-H integration surface.

Figure 14 shows the far field directivity pattern for the two HLDs configurations. The directivity patterns resemble that of a dipole source with the maximum noise radiation in the upstream direction. Deploying the spoiler results in noise level increase at all radiation angles. The largest noise level increase is in the overhead direction.. The maximum noise level increase is $8 \mathrm{~dB}$ and is observed at $\theta=270$ degrees.

The on-body integration surface is constructed to allow the far field noise prediction from individual HLDs components. The far field noise spectra from individual HLDs components at an observer at 270 degrees is shown in Fig. 15. For the conventional HLDs configuration the noise from the flap dominate in the low frequency range with a peak at $100 \mathrm{~Hz}$ corresponding to the vortex shedding frequency at the flap trailing edge. At mid-to-high frequency the noise from the slat is the most dominant. Similar trends are 


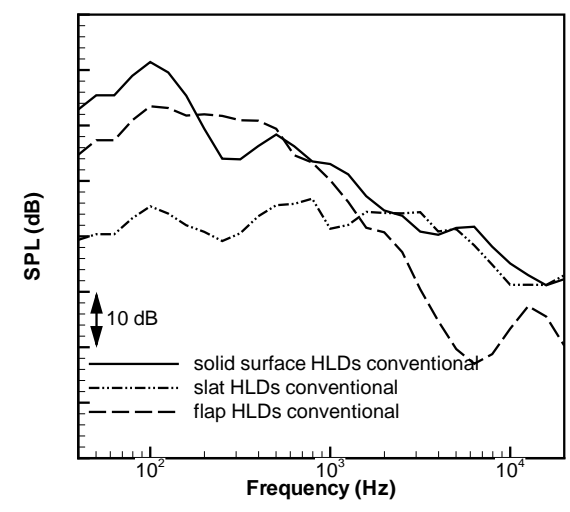

(a) Conventional.

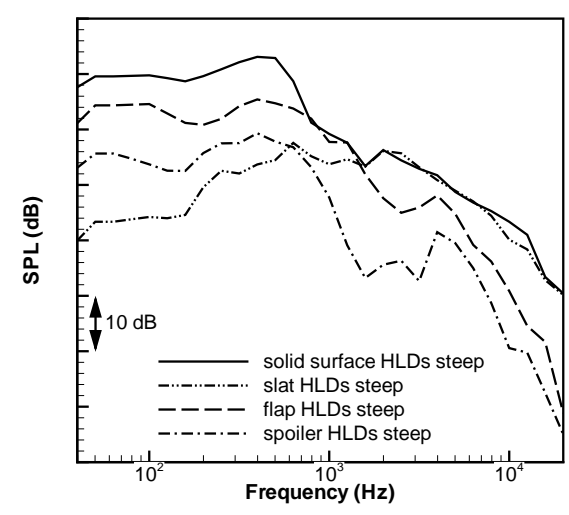

(b) Steep.

Figure 15. Far field pressure spectra for individual HLDs components for an observer at 270 degrees.

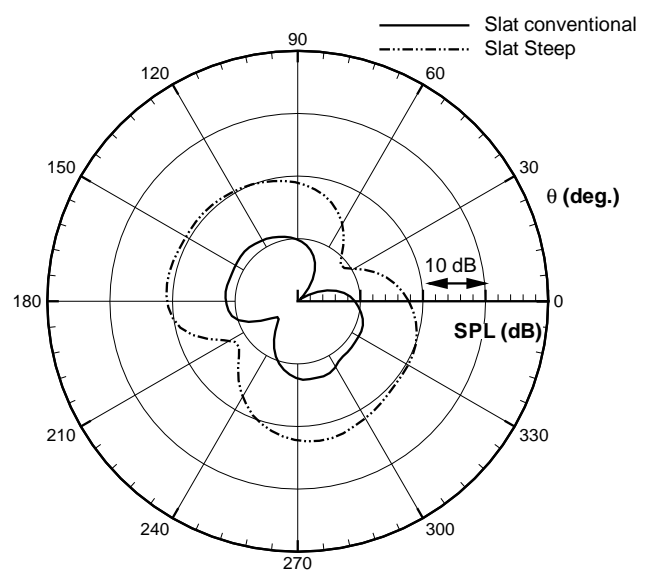

(a) Slat.

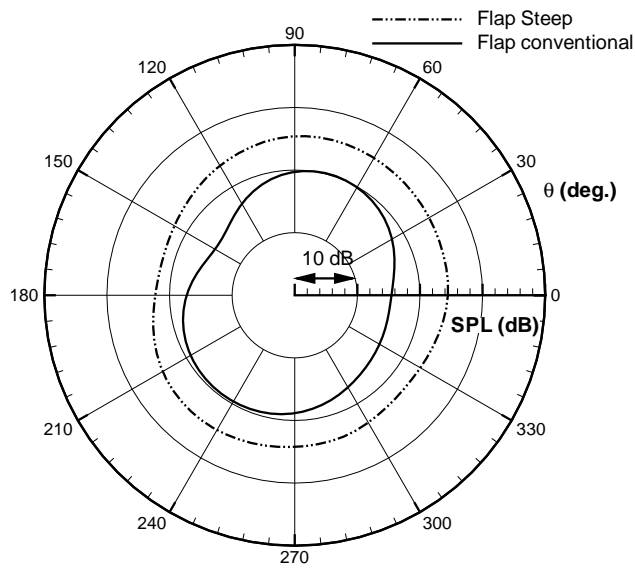

(b) Flap.

Figure 16. Far field directivity from the slat and flap.

observed when the spoielr is deployed. The flap noise is dominant in the low frequencies whereas the slat noise is dominant at mid-to-high frequencies. The noise peak in the slat noise spectrum for the steep HLD configuration (in Fig. 17(a)) at $2.5 \mathrm{kHz}$ corresponds to the vortex shedding frequency from the separation at the main element leading edge. The frequency of the noise peak corresponds to a Strouhal number of 3 based on the slat chord length. According to Dobrzynski and Pott-Pollenske ${ }^{28}$ slat noise spectrum peaks near St $\approx 1-3$ (where the Strouhal number is based on the slat chord). Thus the flow-surface interaction between the vortices from the main element leading edge and the slat trailing edge surface is indeed a source of noise when the spoiler is deflected. The far field noise spectrum from the spoiler has a peak at $4 \mathrm{kHz}$ which is due to the vortex shedding from the spoiler trailing edge. At this frequency the noise contribution from the spoiler is small relative to the noise contribution from the slat. However at low frequency the noise contribution from the spoiler is dominant over the slat noise. For a full 3D wing one would expect the slat noise to dominate over the spoiler noise due to the large surface area of the slat in comparison to the spoiler. In addition for this study the slat trailing edge is idealized as a sharp edge therefore there is no noise contribution from the vortex shedding from the slat trailing edge.

The far field noise directivity from the slat and flap in conventional and steep HLD configurations are shown in Figures 16(a) and 16(b) respectively. The slat far field noise directivity resembles a dipole source with the maximum noise radiation in the direction perpendicular to the slat chord. Similar noise directivity pattern was found by Khorrami and Lockard. ${ }^{14}$ The far field noise directivity from the slat peaks in the overhead position but slightly in the rearward direction. According to Dobrzynski and Pott-Pollenske ${ }^{28}$ slat 


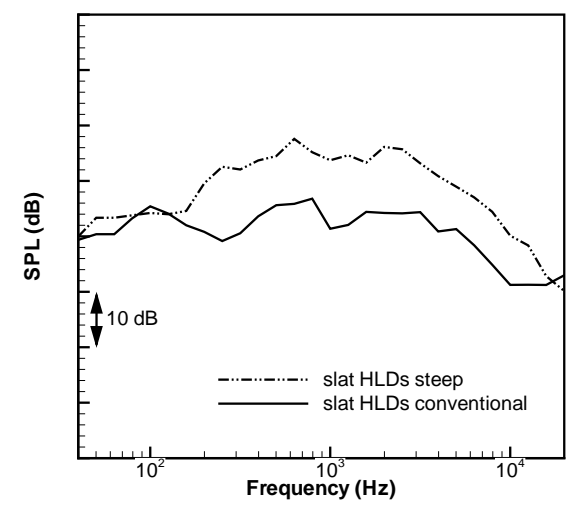

(a) Slat.

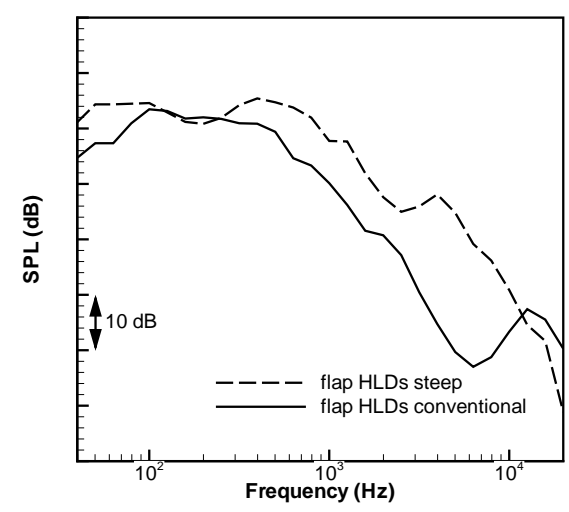

(b) Flap.

Figure 17. Far field pressure spectra from the slat and flap for an observer at 270 degrees.

noise directivity peaks in the rearward direction. The noise level increase at all angles when the spoiler is deployed. The maximum noise level increase is at the overhead position at $\theta=270$ degrees.

The flap far field noise directivity also resembles a dipole source however the similarity is not as obvious. Deploying the spoiler increases the noise level at all angles with the maximum increase directly downstream at $\theta=0$ degrees. The noise increase could be as a result of the interaction of the recirculation flow with the flap surface. The large vortical structures observed in the flow field induce large pressure fluctuations that may radiate as sound.

Figure 17 compares the pressure spectra for the slat and flap for the conventional and steep HLDs configuration. For the slat deploying the spoiler increases the noise level at all frequencies above $150 \mathrm{~Hz}$. At $2.5 \mathrm{kHz}$ the noise level increase by $12 \mathrm{~dB}$ when the spoiler is deployed. Deploying the spoiler increases the flap noise level at all frequencies above $150 \mathrm{~Hz}$. The maximum noise level increase is $20 \mathrm{~dB}$ at $4 \mathrm{kHz}$. The peak in the flap spectrum, for the steep configuration, at $4 \mathrm{kHz}$ is due to the vortex shedding at the spoiler trailing edge. Contours of dilatation (see Fig. 13(b)) showed the acoustic waves from the spoiler trailing edge hitting the flap leading edge.

\section{Summary Remarks}

A computational aeroacoustic analysis of a 2D high-lift configuration in steep approach is performed using a hybrid approach including computational aeroacoustics solution of near flow field and an integral solution of the FW-H equation for far field directivity. The results show the effect of a deployed spoiler on HLD noise and offer an insight into the noise generation mechanisms from HLDs with a deflected spoiler.

The flow field results have shown that deploying the spoiler causes a reduction in circulation around the entire high-lift model. On the slat, the incoming flow incidence at the slat leading edge is reduced. The reduced angle of attack causes an expansion of the slat cove vortex. The roll up of the slat free shear layer into coherent vortices is delayed and occurs further away from the slat cusp when the spoiler is deflected. Also, the flow separates from the main element leading edge and sheds vortices into the gap between the main element and the slat trailing edge. The vortex shedding from the main element leading edge is shown to be a potential noise generation mechanism for the slat in steep configuration. The flow-surface interaction of the shed vortices with the slat sharp trailing edge is a noise source. The vortex shedding at the spoiler trailing edge is also identified as a noise source at $4 \mathrm{kHz}$. Deploying the spoiler also causes the flow to separate early at the flap leading edge. The separated flow forms a large recirculation region above the flap. The interact of the large vortical structures in the flap wake with the flap surface is a possible source of flap noise for this 2D HLD model.

On-body and off-body permeable FW-H integration surfaces are used to predict the far field noise. The prediction from the off-body permeable FW-H integration surface are found to be in error due to the effects of the wake from the flap passing through the surface. Therefore only the on-body FW-H integration surface is used for far field noise prediction. Far field noise directivity and spectra shows that the noise level increases 
at all observer angles and all frequencies above $100 \mathrm{~Hz}$ when the spoiler is deployed. The maximum noise level increase is $8 \mathrm{~dB}$ at $\theta=270$ degrees. Far field pressure spectra of individual HLDs components show that the flap noise is dominant at low frequencies whereas the slat noise is dominant at mid-to-high frequencies. Deploying the spoiler increases the noise from the slat and flap at all frequencies. The noise contribution from the spoiler is significant only at low frequencies.

The study also highlights the deficiency of a 2D simulation. The lack of spanwise extent means the flow is more-energetic in the $2 \mathrm{D}$ simulations that is there are strong vortices that persist over a long period of time, and large separation regions. Therefore the next stage will be performing a 3D simulation of the two configurations to obtain detailed flow physics and to confirm the noise sources and the trends observed in the current 2D study.

\section{Acknowledgments}

The authors would like to thank L.C Chow of Airbus Operations Limited, Bristol, UK and N. Molin of Airbus Operations S.A.S, Toulouse, France for their support in this project. A special thanks to colleagues, especially Dr Ed Peers, at the University of Southampton, for their assistance and insightful discussions.

\section{References}

${ }^{1}$ Crighton, D. G., "Airframe Noise," Aeroacoutics of Flight Vehicles: Theory and Practice, edited by H. H. Hubbard, Vol. 1, NASA RP 1258, 1991, pp. 391-443.

${ }^{2}$ Dobrzynski, W., Nagakura, K., Gehlhar, B., and Buschbaum, A., "Airframe Noise Studies on Wings with Deployed High Lift Devices," AIAA Paper 98-2337, 1998.

${ }^{3}$ Antoine, N. E. and Kroo, I. M., "Aircraft Optimization for Minimal Environmental Impact," Journal of Aircraft, Vol. 41, No. 4, 2004, pp. 790-797.

${ }^{4}$ Clarke, J. B., Ho, N. T., Ren, L., Brown, J. A., Elmer, K. R., Tong, K. O., and Wat, J. K., "Continuous Descent Approach: Design and Flight Test for Louisville International Airport," Journal of Aircraft, Vol. 41, No. 5, 2004, pp. $1054-1066$.

${ }^{5}$ Dobrzynski, W., "Almost 40 Years of Airframe Noise Research - What did we Achieve?" 14th Aeroacoustics Conference, 5-6 May 2008.

${ }^{6}$ Kipersztok, O. and Sengupta, G., "Flight Test of the 747-JT9D for Airframe Noise," Journal of Aircraft, Vol. 19, No. 12, 1982, pp. 1061-1069.

${ }^{7}$ Lee, C. S., Bodapati, S., and Karamcheti, K., "Flow Measurements of an Airfoil with Deflected Spoiler," AIAA Paper 83-0365, 1983.

${ }^{8}$ Bodapati, S., "Unsteady Flow Visualization of Airfoils with Spoilers," Proceedings of the Second Asian Congress of Fluid Mechanics, Science Press, Beijing, China, 1984, pp. 795-800.

${ }^{9}$ Costes, M., Gravelle, A., Philippe, J., Vogel, S., and Triebstein, H., "Investigation of Unsteady Subsonic Spoiler and Flap Aerodynamics," Journal of Aircraft, Vol. 24, No. 9, 1987, pp. 629-637.

${ }^{10} \mathrm{Kim}$, J. H. and Rho, O. H., "Numerical Simulation of Flowfield Around Airfoil with Stationary or Oscillating Spoiler," Journal of Aircraft, Vol. 35, No. 5, 1998, pp. 704-711.

${ }^{11}$ Choi, S., Chang, K., and Ok, H., "Parametric Study of Transient Spoiler Aerodynamics with Two-Equation Turbulence Models," Journal of Aircraft, Vol. 38, No. 5, 2001, pp. 888-894.

${ }^{12}$ Ffowcs-Williams, J. and Hawkings, D., "Sound Generation by Turbulence and Surfaces in Arbitrary Motion," Philosophical Transactions for the Royal Society of London, Series A, Mathematical and Physical Sciences, Vol. 264, No. 1151, 1969, pp. 321-342.

${ }^{13}$ Spalart, P. and Allmaras, S., "A One-equation Turbulence Model for Aerodynamic Flows," AIAA Paper 92-0439, 1992.

${ }^{14}$ Khorrami, M. and Lockard, D., "Effects of Geometric Details on Slat Noise Generation and Propagation," AIAA Paper 2006-2664, May 2006.

${ }^{15}$ Jameson, A., "Time Dependent Calculations Using Multigrid with Applications to Unsteady Flows Past Airfoils and Wings," AIAA Paper 91-1956, 1991.

${ }^{16}$ Ashcroft, G. and Zhang, X., "Optimized Prefactored Compact Schemes," Journal of Computational Physics, Vol. 190, No. 2, 2003, pp. 459-477.

${ }^{17}$ Rizzetta, D., Visbal, M., and Blaisdell, G., "A Time-Implicit High-Order Compact Differencing and Filtering Scheme for Large-Eddy Simulation," International Journal of Numerical Methods in Fluids, Vol. 42, No. 6, 2003, pp. 665-693.

${ }^{18} \mathrm{Kim}, \mathrm{J}$. and Lee, D., "Characteristic Interface Conditions for Multi-block high-order computation on singular structured grid," AIAA Jornal, Vol. 41, No. 12, 2003, pp. 2341-2348.

${ }^{19}$ Takeda, K., Zhang, X., and Nelson, P., "Computational Aeroacoustic simulations of leading-edge slat flow," Journal of Sound and Vibration, Vol. 270, No. 3, 2004, pp. 559-572.

${ }^{20}$ Farassat, F. and Succi, G., "The Prediction of Helicopter Rotor Discrete Frequency Noise," Vertica, Vol. 7, No. 4, 1983, pp. 309-320.

${ }^{21} \mathrm{Ma}$, Z. and Zhang, X., "Numerical Investigation of Broadband Slat Noise Attenuation with Acoustic Liner Treatment," AIAA Jornal, Vol. 47, No. 12, 2009, pp. 2812-2820. 
${ }^{22}$ Ashcroft, G. and Zhang, X., "A Computational Investigation of the Noise Radiated by Flow-Induced Cavity Oscillations," AIAA Paper 2001-0512, 2001.

${ }^{23}$ Khorrami, M., Singer, B., and Lockard, D., "Time accurate simulations and acoustic analysis of slat free-shear-layer:Part II," AIAA Paper 2002-2579, 2002.

${ }^{24}$ Terracol, M., Manoha, E., Herrero, C., Labourasse, E., Rodennet, S., and Sagaut, P., "Hybrid Methods for Airframe Noise Numerical Prediction," Theoretical Computational Fluid Dynamics, Vol. 19, 2005, pp. 197-227.

${ }^{25}$ Takeda, K., Zhang, X., Ashcroft, G., and Nelson, P., "Unsteady Aerodynamics of Flap Cove Flow in a High-Lift Device Configuration," AIAA Paper 2001-706, 2001.

${ }^{26}$ Bogey, C., Bailly, C., and Juve, D., "Computation of Flow Noise Using Source Terms in Linearized Euler's Equations," AIAA Journal, Vol. 40, No. 2, 2002, pp. 235-243.

${ }^{27}$ Casper, J. H., Lockard, D. P., Khorrami, M. R., and Street, C. L., "Investigation of Volumetric Sources in Airframe Noise Simulations," AIAA Paper 2004-2805, 2004.

${ }^{28}$ Dobrzynski, W. and Pott-Pollenske, M., "Slat Noise Source Studies for Farfield Noise Prediction," AIAA Paper 2001-2158, 2001. 